\title{
Primeiro registro de Anopheles (Kerteszia) homunculus Komp (Diptera, Culicidae) no Estado do Espírito Santo, Brasil
}

\author{
Maria Anice Mureb Sallum ${ }^{1}$, Paulo Roberto Urbinatti ${ }^{1}$, Rosely dos Santos Malafronte ${ }^{2,3}$, \\ Helder Ricas Resende ${ }^{4}$, Crispim Cerutti $\mathrm{Jr}^{2,5}$ \& Delsio Natal ${ }^{1}$
}

\begin{abstract}
'Faculdade de Saúde Pública da Universidade de São Paulo, Departamento de Epidemiologia. masallum@usp.br; urbinati@usp.br; natal@usp.br ${ }^{2}$ Faculdade de Medicina da Universidade de São Paulo, Departamento de Doenças Infecciosas e Parasitárias.

${ }^{3}$ Instituto de Medicina Tropical, Laboratório de Protozoologia. rmalafronte@usp.br

${ }^{4}$ Secretaria de Estado da Saúde, Governo do Estado do Espírito Santo. heldericas@hotmail.com

${ }^{5}$ Universidade Federal do Espírito Santo, Departamento de Ciências da Saúde e Unidade de Medicina Tropical, Departamento de Medicina Social. fil.cris@terra.com.br
\end{abstract}

\begin{abstract}
First record of Anopheles (Kerteszia) homunculus Komp (Diptera, Culicidae) in the state of Espírito Santo, Brazil. In a field collecting trip carried out in the State of Espírito Santo, Brazil, in January 2006, as part of a study on Plasmodium transmission, Anophelinae larvae were collected in bromeliads. The immatures were raised in the laboratory until adult stage, and thus adults male and female and their associated larval and pupal exuviae were mounted and identified. Consequently, it was verified that two specimens belong to Anopheles (Kerteszia) homunculus Komp, 1937. This is the first record of that Kerteszia species in Espírito Santo. The present finding shows the importance of additional studies aiming to establish the geographical distribution of An. homunculus in addition to the taxonomic status and the epidemiological importance of the species in the dynamics of malaria transmission in areas of Atlantic Forest.
\end{abstract}

KEYWORDS. Distribution; Plasmodium vector; Mata Atlântica.

RESUMO. Primeiro registro de Anopheles (Kerteszia) homunculus Komp (Diptera, Culicidae) no Estado do Espírito Santo, Brasil. Em pesquisa de campo realizada no interior do Estado do Espírito Santo, Brasil, em janeiro de 2006, como parte de projeto sobre a transmissão de Plasmodium, foram coletadas larvas de anofelinos em bromélias. Os imaturos foram mantidos no laboratório até a obtenção dos adultos machos e fêmeas associados com as exúvias das larvas e das pupas, para serem identificados. Conseqüentemente, verificou-se que dois espécimes pertenciam a Anopheles (Kerteszia) homunculus Komp, 1937. Este é o primeiro registro dessa espécie de Kerteszia no Espírito Santo. O encontro evidencia a importância de estudos adicionais de modo a estabelecer a distribuição geográfica do An. homunculus, bem como o status taxonômico e a importância epidemiológica da espécie na dinâmica da transmissão da malária em áreas de Mata Atlântica.

PALAVRAS-CHAVE. Distribuição; Mata Atlântica; Vetores de plasmódio.

A malária no Brasil é considerada relevante, principalmente na Região Amazônica onde é referenciada como endêmica. Fora desse território há constantes relatos de casos importados, sejam provenientes da área endêmica do país ou do exterior. Em todo o território extra-amazônico há riscos de transmissão, sempre que ocorram: condições ecológicas satisfatórias, introdução de indivíduos gametóforos, elevada densidade de anofelíneos competentes, entre outros requisitos.

A transmissão autóctone de malária extra-amazônica ocorre em vários estados brasileiros, principalmente naqueles associados ao Sistema Serra do Mar. Nessa região manifestase geralmente em condição hipoendêmica, com casos isolados ou pequenos surtos. Tal situação mantém-se principalmente devido à marcante presença de mosquitos do subgênero Kerteszia, grupo de anofelíneos que se desenvolvem nas águas acumuladas nas axilas de bromélias (exceto $A n$. bambusicolus que se desenvolve em internódios de bambu).

No Estado do Espírito Santo, casos de malária autóctone ocorrem predominantemente nas áreas montanhosas recobertas pela Mata Atlântica, ao longo do semicírculo que tem por centro a cidade de Vitória. Estão incluídos na região os municípios de Santa Leopoldina, Santa Teresa, Santa Maria do Jetibá, Domingos Martins, Viana, Marechal Floriano, Alfredo Chaves e Vargem Alta. A área com casos caracteriza-se por altitudes ao redor de oitocentos metros acima do nível do mar e coincide com a distribuição de Anopheles (Kerteszia) cruzii Dyar \& Knab no Estado, fato que sugere ser esse anofelíneo o responsável pela manutenção da transmissão autóctone de malária Rezende et al. (2005).

Nas regiões da Mata Atlântica, An. cruzii e Anopheles (Kerteszia) bellator Dyar \& Knab foram muitas vezes incriminadas como as espécies responsáveis pela transmissão de Plasmodium vivax Grassi \& Feletti . Os registros da literatura indicam que An. cruzii é usualmente o vetor encontrado com as maiores freqüências nos locais onde são diagnosticados os casos de malária (Rachou 1958; Forattini 1962; Forattini et al. 1986). Dentre outros anofelíneos desse subgênero associados ao Sistema Serra do Mar, destaca-se o An. 
homunculus, porém, no Brasil, a espécie nunca foi registrada em latitudes inferiores às do Estado de São Paulo (Zavortink 1973).

O An. homunculus foi descrito por Komp (1937) a partir de espécimes coletados em Restrepo ( $\mathrm{S}+4^{\circ} 10^{\prime} 1.20^{\prime \prime} \mathrm{W}-73^{\circ} 25^{\prime}$ 1.20"), Departamento del Meta, Colômbia. As formas imaturas desenvolvem-se na água acumulada nas axilas de bromélias epífitas e terrestres e as fêmeas adultas podem se alimentar de sangue humano. An. homunculus é vetor importante de plasmódios humanos em áreas do sudeste do Brasil e em Trinidad (Forattini 1962). Pittendrigh (1948, 1950a, 1950b, 1950c) comparou An. bellator e An. homunculus presentes em Trinidad e demonstrou que a população desta habita áreas onde a umidade relativa do ar é maior. Conseqüentemente, o An. homunculus é comumente encontrado em regiões mais úmidas e nos níveis mais baixos na floresta. Aspecto ecológico semelhante foi observado no sudeste do Brasil, onde esta espécie está presente em áreas de floresta tropical úmida com vegetação densa (Aragão 1964). No entanto, sua distribuição geográfica não é bem conhecida. Registros publicados na literatura demonstram que a espécie foi encontrada em áreas ao leste dos Andes na Colômbia e Bolívia, Trinidad, sul do Brasil, Guianas, Peru, Suriname e Venezuela (Guimarães 1997). No Brasil, vale assinalar os registros de Aragão (1964) e de Forattini et al. (1970) sobre a presença de An. homunculus, em Santa Catarina e no Paraná, respectivamente. A existência de poucos registros desta espécie no Brasil pode estar associada à dificuldade em sua identificação. As fêmeas adultas do $A n$. homunculus são morfologicamente semelhantes aquelas de An. cruzii. As duas espécies podem ser diferenciadas por caracteres presentes nos palpos maxilares e pela coloração dos tergitos abdominais. Além disso, estruturas da terminália masculina são distintas e permitem o reconhecimento de $A n$. homunculus. Caracteres das pupas e das larvas de quarto estádio também permitem a identificação de espécimes de $A n$. homunculus (Forattini 1962; Zavortink 1973). Forattini (1962) assinala que a coloração arroxeada das formas imaturas de $A n$. homunculus permite o diagnóstico da espécie.

Como parte de projeto de pesquisa que objetiva estudar a dinâmica de transmissão de Plasmodium na região serrana do Estado do Espírito Santo, em trabalho de campo destinado à obtenção de material para estudos taxonômicos, larvas de anofelíneos foram coletadas e posteriormente identificadas como An. homunculus. Relata-se na presente publicação a importância desse encontro.

No período de 16 a 18 de janeiro de 2006, coletaram-se culicídeos no Estado do Espírito Santo, nos municípios de Linhares, Sooretama e Jaguaré, no Vale do Rio Doce, situados fora da área de transmissão de malária e no município de Santa Teresa, na região serrana, onde casos autóctones dessa doença foram notificados. Destaca-se a coleta feita no município de Santa Teresa, localidade de Valsugana (S 19 57' 58,4" W $40^{\circ}$ $34^{\prime} 45,2^{\prime \prime}$ ), por ter sido o local onde foram coletadas as larvas que motivaram a presente divulgação.

A localidade de Valsugana caracteriza-se por terrenos acidentados, com marcante presença antrópica, predominantemente de descendentes de imigrantes italianos, que ocupam os fundos de vale e partes das encostas das colinas. A atividade rural é de subsistência, dominando a cultura de café. As áreas de topografia mais íngreme e as partes mais altas estão preservadas e cobertas por resíduos florestal típicos
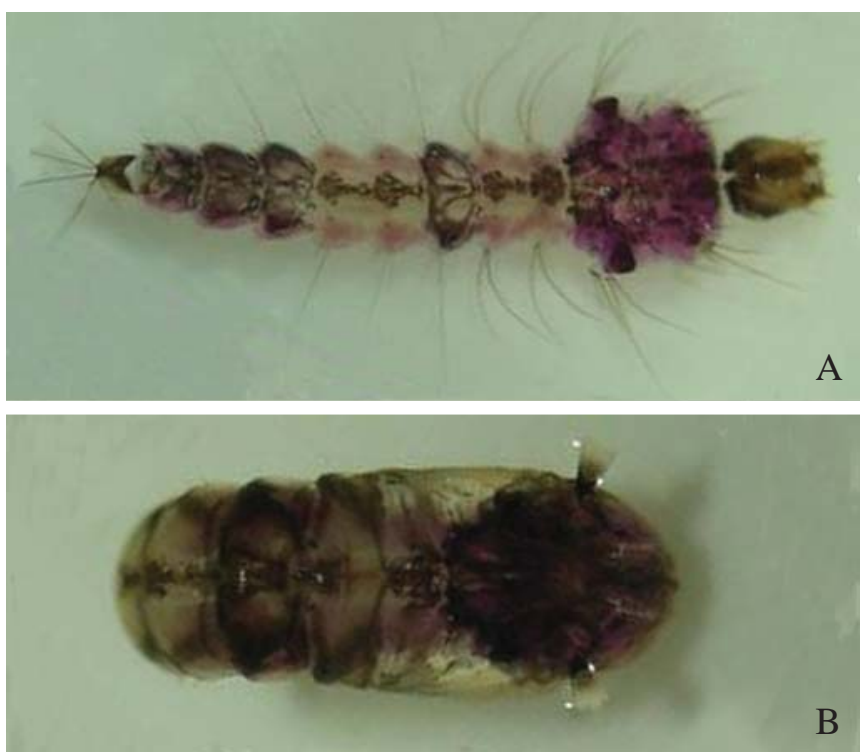

Fig. 1. Formas imaturas de Anopheles (Kerteszia) homunculus.

A - Larva de quarto estádio em fase de pré-pupa. B - Pupa.

da Mata Atlântica regional, com elementos arbóreos que comportam bromélias epífitas.

As formas imaturas foram coletadas a partir de bromélias aderidas aos galhos de uma árvore caída. O líquido foi extraído com o auxílio de bomba de sucção manual tendo as bromélias sido preservadas de mutilações. O conteúdo foi transferido para frascos, os quais foram conduzidos ao laboratório. $\mathrm{O}$ ponto de coleta corresponde a uma mata residual relativamente preservada situada na parte mais elevada de uma colina.

No laboratório as larvas foram mantidas na água coletada nas bromélias, até a emergência de adultos. Obtiveram-se amostras de adultos de ambos os sexos, associados às exúvias da larva de quarto estádio e da pupa. As exúvias das larvas de quarto estádio e das pupas foram processadas e montadas em Bálsamo do Canadá entre lâmina e lamínula. Os adultos e a terminália do macho foram processados e montados. Os espécimes foram depositados na Coleção de Referência da Faculdade de Saúde Pública da Universidade de São Paulo.

O conteúdo líquido coletado nas bromélias continha quatro larvas de anofelíneos, identificadas enquanto vivas como pertencentes ao subgênero Kerteszia. Duas dessas larvas apresentavam tonalidade avermelhada típica de imaturos de An. cruzii e as outras duas, arroxeadas, indicando tratar-se de An. homunculus (Fig. 1a, b). Segundo Forattini (1962), essa cor é conferida por grânulos de pigmentos depositados no tecido subcutâneo. As figuras 1a e 1b ilustram uma das larvas coletadas e a pupa correspondente, evidenciando a tonalidade característica. Dessa maneira, foi possível identificar as espécies dos quatro espécimes de Kerteszia utilizando os diversos estádios de desenvolvimento, indicando pela primeira vez a presença de An. homunculus no Espírito Santo.

Considerava-se que no Brasil, a distribuição de $A n$. homunculus estendia-se do norte do Rio Grande do Sul ao sul do Estado de São Paulo (Aragão 1964). Estudo de fauna de mosquitos de bromélias em Peruíbe (S 24 19' 0" S 470 0' W"), São Paulo comprovou a presença dessa espécie em área da Serra do Mar paulista (Seto 1992). 
Sem desconsiderar a ampla distribuição do An. homunculus na América do Sul (Guimarães 1997), a identificação desse anofelíneo procedente da região serrana do Estado do Espírito Santo expande no Brasil sua distribuição geográfica mais ao norte, da latitude de $24^{\circ} \mathrm{S}$ para $19^{\circ} \mathrm{S}$, na faixa correspondente à Serra do Mar.

An. homunculus foi considerada como participante na epidemiologia da malária, tanto na área de "bromélia-malária" no Brasil, como em Trinidad, porém outros mosquitos como An. bellator e An. cruzii foram reconhecidos como vetores primários (Zavortink 1973; Gadelha 1994). Na região serrana do Espírito Santo devido à presença confirmada de An. cruzii que se sobrepõe à área com casos de malária é pertinente a possibilidade de ser esse anofelíneo o vetor (Rezende et al. 2005). Ampliando essa discussão, vale assinalar que nada se pode afirmar, até o momento, sobre o envolvimento do An. homunculus na dinâmica da transmissão da malária na região de Santa Teresa e outros municípios afetados. No entanto, a presença desse mosquito torna evidente a necessidade de estudos para se verificar tanto a distribuição geográfica como caracterizar aspectos da ecologia e do papel epidemiológico desse anofelíneo no Espírito Santo.

Como An. cruzii e An. homunculus fazem parte de um complexo de espécies morfologicamente semelhantes é possível que as identificações referidas à primeira espécie em Santa Teresa (Rezende et al. 2005) possam contemplar indivíduos da segunda espécie que foram identificados erroneamente. Essa suposição demonstra a necessidade de utilizar caracteres morfológicos dos diferentes estágios de desenvolvimento para a identificação das espécies de Culicidae que compõe a fauna de determinada localidade. Obviamente, a identificação correta das espécies é de fundamental importância tanto para o reconhecimento dos vetores envolvidos na transmissão de patógenos como para elaboração de estratégias de controle.

$\mathrm{O}$ encontro aqui relatado evidencia a necessidade de estudos sistemáticos detalhados que incluam tanto a caracterização morfológica quanto a molecular de $A n$. homunculus, como aqueles realizados para An cruzii. Nesse sentido vale assinalar os estudos empregando sequiências de nucleotídeos do segundo espaçador interno transcrito (ITS2) do DNA ribossômico nuclear (Malafronte et al. 2007), padrão isoenzimático (Carvalho-Pinto \& Lourenço-de-Oliveira 2004) e de cromossomos politênicos (Ramirez \& Dessen 1994, 2000a, 2000b; Ramirez et al. 1994) de exemplares de An. cruzii coletados em distintas localidades do Brasil. Estudo empregando marcadores moleculares (PCR-RAPD e RFLP da região ITS2 e parte dos genes $5.8 \mathrm{~S}$ e 28S) para An. homunculus foi realizado por Calado \& Navarro-Silva (2005) com espécimes coletados em São Francisco do Sul (Estado de Santa Catarina). Os exemplares de An. homunculus foram comparados com outros de An. cruzii obtidos em localidades diversas dos estados do Paraná, Santa Catarina e São Paulo. Dessa maneira, demonstrou-se ser possível a separação das duas espécies.

Agradecimentos. A Fundação de Amparo à Pesquisa do Estado de São Paulo pelo financiamento dos estudos (Fapesp) processos $n^{\circ} 05 /$
53973-0, 03/07631-4 e Conselho Nacional de Desenvolvimento Científico e Tecnológico (CNPq) processo $\mathrm{n}^{\circ}$. 472485/2006-7).

\section{REFERÊNCIAS}

Aragão, M. B. 1964. Distribuição geográfica e abundância das espécies de Anopheles (Kerteszia) (Diptera: Culicidae). Revista Brasileira de Malariologia e Doenças Tropicais 16: 73-109.

Calado D. C. \& M. A. Navarro-Silva. 2005. Identificação de Anopheles (Kerteszia) cruzii Dyar \& Knab e.Anopheles (Kerteszia) homunculus Komp (Diptera, Culicidae, Anophelinae) através de marcadores moleculares (RAPD e RFLP). Revista Brasileira de Zoologia 22: $1127-1133$.

Carvalho-Pinto, C. J. \& R. Lourenço-de-Oliveira. 2004. Isoenzimatic analysis of four Anopheles (Kerteszia) cruzii (Diptera: Culicidae) populations of Brazil. Memórias do Instituto Oswaldo Cruz 99: 471-475.

Forattini, O. P. 1962. Entomologia Médica. Culicini: Culex, Aedes e Psorophora. São Paulo, Edgard Blucher, 506 p.

Forattini, O. P.; E. X. Rabello \& M. D. Cotrim. 1970. Catálogo das coleções entomológicas da Faculdade de Saúde Pública da Universidade de São Paulo (1 ${ }^{\mathrm{a}}$. Série). Culicidae. Revista de Saúde Pública 4: $1-$ 100 .

Forattini, O. P.; A. C. Gomes; D. Natal \& J. L. F. Santos. 1986. Observações sobre a atividade de mosquitos Culicidae em matas primitivas da planície e perfis epidemiológicos de vários ambientes do Vale do Ribeira, São Paulo, Brasil. Revista de Saúde Pública 20: 178-203.

Gadelha, P. 1994. From "forest malaria" to "bromeliad malaria": a casestudy of scientific controversy and malaria control. Parasitologia 56: $175-195$.

Guimarães, J. H. 1997. Systematic database of Diptera of de Americas South of the United States (family Culicidae). São Paulo, Editora Plêiade / Fapesp, 286 p.

Komp, W. H. W. 1937. The species of the subgenus Kerteszia of Anopheles (Diptera: Culicidae). Annals of the Entomological Society of America 30: 492-529.

Pittendrigh, C. S. 1948. The bromeliad-Anopheles-malaria complex in Trinidad. I - The bromeliad flora. Evolution 2: 58-89.

Pittendrigh, C. S. 1950a. The quantitative evaluation of Kerteszia breeding grounds. American Journal of Tropical Medicine 30: 457-468.

Pittendrigh, C. S. 1950b. The climate divergence of An. bellator and An. homunculus. Evolution 4: 43-63.

Pittendrigh, C. S. 1950c. The ecotopic specialization of Anopheles homunculus; and its relation to competition with A. bellator. Evolution 4: 64-78.

Rachou, R. G. 1958. Anofelinos no Brasil: comportamento das espécies vetoras de malária. Revista Brasileira de Malariologia e Doenças Tropicais 10: 145-81.

Ramirez, C. C. L. \& E. M. Dessen. 1994. Cytogenetics analysis of a natural population of An.cruzii. Revista Brasileira de Genética 17: 41-46.

Ramirez, C. C. L. \& E. M. Dessen. 2000a. Chromosome differentiated populations of Anopheles cruzii: evidence for a third sibling species. Genética 108: 73-80.

Ramirez, C. C. L. \& E. M. Dessen. 2000b. Chromosomal evidence for sibling species of the malaria vector Anopheles cruzii. Genome 43: $143-51$.

Ramirez, C. C. L.; E. M. B. Dessen \& P. A. Otto 1994. Inversion polymorphism in a natural population of Anopheles cruzii. Caryologia 47: $121-130$.

Rezende, H. R.; C. Cerutti-Jr \& C. B. Santos. 2005. Aspectos atuais da distribuição geográfica de Anopheles (Kerteszia) cruzii Dyar \& Knab, 1908 no Estado do Espírito Santo, Brasil. Entomología y Vectores 12: $123-26$.

Seto, M. I. 1992. Ocorrência de mosquitos (Diptera: Culicidae) em bromélias da localidade de Aldeia dos Indios, área endêmica de malária no município de Peruíbe (SP), no período de julho de 1985 a julho de 1987. (Dissertação apresentada ao Instituto de Ciências Biomédicas da Universidade de São Paulo, para obtenção do título de Mestre em Parasitologia). 127 p.

Zavortink, T. J. 1973. Mosquito studies (Diptera: Culicidae) XXIX. A review of the subgenus Kerteszia of Anopheles. Contribution of the American Entomological Institute 9: 1-54. 